\title{
Donepezil-Induced Onset of Rheumatoid Arthritis: Case Report and Literature Review
}

Xiaohong Liu, Jianzhong Zhu, Dan Liu and Xiaowei Liu*

Wuxi mental health center, Wuxi, Jiangsu, China

\begin{abstract}
Donepezil has been approved for treatment of Alzheimer's disease. The common adverse reactions of donepezil in the treatment of Alzheimer's disease include diarrhea and nausea. However, there are also some other rare clinical cases been reported on administration of donepezil, such as mania and delirious behavior. Here, we report a patient with Alzheimer's disease who was induced the onset of rheumatoid arthritis upon treatment with donepezil
\end{abstract}

Keywords: Donepezil; Rheumatoid arthritis; Cholinergic; Inflammation

\section{Introduction}

Donepezil, rivastigmine and galantamine, three acetylcholinesterase/ cholinesterase (AChE/ChE) inhibitors on the market are considered the standard treatment of the mild-moderate stage of Alzheimer's disease (AD) [1-3]. By inhibition of AChE/ChE, the enzymes degrading acetylcholine in the synaptic cleft, they enhance the cholinergic transmission and slow down of the acetylcholine (ACh) catabolism which makes acetylcholine more concentrated [4].

In a systematic review and meta-analysis, it has been found that on average across all included trials, $76 \%$ of patients administered with donepezil, rivastigmine and galantamine reported at least one adverse event. The most frequently reported adverse events associated with donepezil were diarrhea $12 \%$, nausea $11 \%$, vomiting $7 \%$, dizziness $8 \%$, and weight loss $7 \%$ [3]. As these adverse events are usually mild and transient, there are also some other clinical cases been reported on administration of donepezil, such as mania and delirious behavior $[5,6]$.

To the best of our knowledge, donepezil induced onset of rheumatoid arthritis (RA) has not been reported previously. Here, we present a case of onset of RA associated with donepezil administration in a patient of $\mathrm{AD}$. Fortunately, withdrawal of donepezil effectively attenuated the symptoms of RA. The patient and her family provided informed consent for publication.

\section{Case Presentation}

A 78-year-old Chinese woman presented to our outpatient geriatric psychiatry clinic for evaluation of "a gradual onset of cognitive and behavioral impairment over four years". The patient had a previous history of RA for about ten years and diabetes mellitus for about three years, with no prior history of mental illness. The family members of the patient have been complaining of her memory impairment and behavioral imbalance 4 years prior, including misplacing personal belongings, repetitive questions and conversations, getting lost on a familiar route, difficulty thinking of common words while speaking, social withdrawal and agitation. Examination of her mental status revealed of impairment in recalling of recently learned information, deficits in word-finding, object agnosia, impaired reasoning, judgment and problem solving.

We reviewed her psychiatric history and found that a diagnosis of $\mathrm{AD}$ was made 4 years prior. For the present episode, the diagnosis of
$\mathrm{AD}$ was made by a psychiatrist in our hospital according to the fourth edition of the Diagnostic and Statistical Manual of Mental Disorders [7]. She was treated with donepezil at a dose of $5 \mathrm{mg} /$ day for variable periods during the three previous psychiatric hospitalizations. On admission, she was mentally aware. Her Mini Mental State Examination score was 2 and Hachinski ischemic index (HIS) score was 3. She displayed no neurological signs or symptoms. Findings of laboratory tests were normal. Computed tomography of the brain revealed brain atrophy and white matter change. However, electroencephalography findings were normal.

She was administered donepezil at a dose of $5 \mathrm{mg} /$ day for improvement of the cognitive function. 3 days later, however, the patient complained of low fever, multiple joint pain and swelling. The affected joints were small joints of hands and wrist joints. The joint pain and swelling made her difficult in holding things with her hands. Physical examination revealed redness, increased skin temperature and significant swelling of her small joints of hands and wrist joints. Several rheumatoid tubercles were also noticed on her small joints of hands. Other systemic examinations were unremarkable. $\mathrm{X}$ rays of the hands and wrist joints showed osteoporosis and articular destructive changes entirely consistent with RA. Laboratory investigation showed Hb- 96 g/l, WBC- 95,000/cmm (N-62\%, L-32\%), platelet- 320,000/cmm, CRP$58.8 \mathrm{mg} / \mathrm{l}$ (normal reference- $<9 \mathrm{mg} / \mathrm{l}$ ), ESR- $20 \mathrm{~mm}$ in 1 st hour, RF 28.5 IU/L(baseline 14IU/L), Ig A- $5.30 \mathrm{~g} / \mathrm{L}$ (normal reference $0.70-4.00 \mathrm{~g} / \mathrm{L}$ ), Ig G- $22.70 \mathrm{~g} / \mathrm{L}$ (normal reference 7.00-16.00 g/L) and ASO- 277.6IU/L (baseline 200IU/L). These results suggested an auto-immune disorder and we excluded osteoarthritis.

She was treated symptomatically with NSAID (indomethacin) for 2 weeks. Her symptoms improved within a week except joint swelling. We reviewed her medical history again and noticed that every time she was prescribed donepezil, onset of RA was induced, and interestingly, the swellings of the hands and wrist joints would not completely resolve

*Corresponding author: Xiaowei Liu, Wuxi Mental Health Center, Wuxi, Jiangsu, China, Tel:86-510-83219395; E-mail: 413138889@qq.com

Received January 29, 2015; Accepted March 10, 2015; Published March 17 2015

Citation: Liu XH, Zhu JZ, Liu D, Liu XW (2015) Donepezil-Induced Onset of Rheumatoid Arthritis: Case Report and Literature Review. J Psychiatry 18: 266 doi: 10.4172/2378-5756.1000266

Copyright: @ $2015 \mathrm{Liu} \mathrm{XH}$, et al. This is an open-access article distributed under the terms of the Creative Commons Attribution License, which permits unrestricted use, distribution, and reproduction in any medium, provided the original author and source are credited 
until donepezil been withdrawn. So we withdrew donepezil, and the swellings of the hands and wrist joints resolved a week later.

\section{Discussion}

The common adverse effects of donepezil are, as mentioned above, diarrhea, nausea, vomiting, dizziness and weight loss [3]. Usually, these adverse events are mild and transient. However, there are also some psychotic symptoms, such as mania and delirious behavior, have been reported to be associated donepezil $[5,6]$. But donepezil induced onset of RA has not been reported previously. We reviewed literature and found that studies assessing the effect of AChE inhibition upon inflammation have yielded conflicting results.

On the one hand, ACh has been demonstrated anti-inflammatory functions [8-13]. In a lipopolysaccharides (LPS)-stimulated human macrophage cultures experiment, Borovikova et al. found that ACh significantly attenuated the release of TNF, IL- $1 \beta$, IL- 6 and IL-18, but not the anti-inflammatory cytokine IL-10, and they further demonstrated that during lethal endotoxemia in rats, direct electrical stimulation of the peripheral vagus nerve inhibited TNF synthesis in liver, attenuated peak serum TNF amounts, and prevented the development of shock [8]. Similarly, Wang et al. reported that ACh inhibits high mobility group box 1 (HMGB1) release from human macrophages and nicotine, a selective cholinergic agonist, is more efficient than ACh and inhibits HMGB1 release induced by either endotoxin or TNF- $\alpha$. In vivo, treatment with nicotine attenuates serum HMGB1 levels and improves survival in experimental models of sepsis [9]. In addition, recent studies have demonstrated that the use of AChE inhibitors suppress systemic inflammation and enhance the survival of animals exposed to LPS $[12,13]$. Treatment of galanthamine, another AChE/ChE inhibitor, decreased the level of circulating TNFain rats with LPS-induced peritonitis with sham operation, but could not decrease the TNF- $\alpha$ level in rats with LPS-induced peritonitis with vagotomy [12]. Peripheral administration of galantamine significantly reduced serum TNF-alevels, and protected against lethality during murine endotoxemia, while administration of a centrally-acting muscarinic receptor antagonist abolished the suppression of TNF-aby galantamine, which showed that inhibition of brain AChE suppresses systemic inflammation [13].

On the other hand, some scientists got conflicting results [14-16] Inhibition of AChE induced by a subchronic exposure to paraoxon was reported to render animals more resistant to infection by a virulent strain of Salmonella enterica serovar Typhimurium, a Gram-negative enteric pathogen. Inhibition of AChE enabled the animals to mount a more effective inflammatory anti-microbial response, and to secrete higher levels of IL-12 [14]. Hofer and his colleagues observed that intraperitoneal injections of either nicotine or the AChE inhibitors physostigmine or neostigmine significantly reduced lethality after sepsis induced by cecal ligation [15]. In a mice model of septic shock induced by Escherichia coli, the simultaneous administration of neostigmine and endotoxin decreased interstitial inflammation in the lungs, vacuolar degeneration in the liver, and total liver injury [16].

In this case, we were compelled to speculate that the onset of RA was associated with donepezil administration for the following reasons: (i) the symptoms of RA appeared three days after administration of donepezil; (ii) the swellings of the hands and wrist joints resolved a week later after withdrawal of donepezil; and (iii) every time she was prescribed donepezil in her three periods of hospitalization, onset of RA was induced and the swellings of the hands and wrist joints would not completely resolve until donepezil been withdrawn.
In conclusion, this case report suggested that donepezil could induce onset of RA upon treatment of $\mathrm{AD}$. In a study which reviewed 7490 hospital discharges of elderly patients seeking concomitant diagnoses of $\mathrm{RA}$ and $\mathrm{AD}$, it was found that the rate of such occurrence was $0.39 \%$, or six to twelve times lower than would have been predicted (assuming independence of the two diagnoses) by the product of the rates for the individual diseases [17]. As there are growing numbers of $\mathrm{AD}$ patients worldwide, there would also be lots of patients of RA would eventually develop $\mathrm{AD}$ in later life. In these patients, the onset of RA would likely influence their quality of life and physicians should be cautious in prescribing it in patients with RA.

\section{References}

1. Doody RS, Dunn JK, Clark CM (2001) Chronic donepezil treatment is associated with slowed cognitive decline in Alzheimer's disease. Dement Geriatr Cogn Disord 12: 295-300.

2. Clegg A, Bryant J, Nicholson T (2001) Clinical and cost-effectiveness of donepezil, rivastigmine and galantamine for Alzheimer's disease: a rapid and systematic review. Health Technol Assess 5: 1-137.

3. Hansen RA, Gartlehner G, Webb AP, Morgan LC, Moore CG, et al. (2008) Efficacy and safety of donepezil, galantamine, and rivastigmine for the treatment of Alzheimer's disease: a systematic review and meta-analysis. Clin Interv Aging 3: 211-225.

4. Molino I, Colucci L, Angiola MF, Traini E, Amenta F (2013) Efficacy of Memantine, Donepezil, or Their Association in Moderate-Severe Alzheimer's Disease: A Review of Clinical Trials. ScientificWorldJournal 2013: 925702.

5. Wicklund S, Wright M (2012) Donepezil-Induced Mania [J]. Journal of Neuropsychiatry and Clinical Neurosciences 24: 10027.

6. Mollazadeh-Moghaddam K, Jamali A, Adili-Aghdam F (2013) Delirium Associated with Donepezil in a Patient with Alzheimer's Disease: a Case Report [J]. Iran J Psychiatry 8: 59

7. American Psychiatric Association (1994) Diagnostic and Statistical Manual of Mental Disorders (4thedn). Washington, DC: American Psychiatric Association.

8. Borovikova LV, Ivanova S, hang M, Yang H, Botchkina GI, et al. (2000) Vagus nerve stimulation attenuates the systemic inflammatory response to endotoxin. Nature 405: 458-462.

9. Wang $\mathrm{H}$, Liao $\mathrm{H}$, Ochani $M$, Justiniani M, Lin X, et al. (2004) Cholinergic agonists inhibit HMGB1 release and improve survival in experimental sepsis. Nat Med 10: 1216-1221.

10. Pavlov VA, Tracey KJ (2004) Neural regulators of innate immune responses and inflammation. Cell Mol Life Sci 61: 2322-2331.

11. Pavlov VA, Tracey KJ (2006) Controlling inflammation: The cholinergic antiinflammatory pathway. Biochem Soc Trans 34: 1037-1040.

12. Liu ZH, Ma YF, Wu JS, Gan JX, Xu SW, et al. (2010) Effect of cholinesterase inhibitor galanthamine on circulating tumor necrosis factor alpha in rats with lipopolysaccharide-induced peritonitis. Chin Med J 123: 1727-1730.

13. Pavlov VA, Parrish WR, Rosas-Ballina M, Ochani M, Puerta M, et al. (2009) Brain acetylcholinesterase activity controls systemic cytokine levels through the cholinergic anti-inflammatory pathway. Brain Behav Immun 23: 41-45.

14. Fernandez C, Lorke MJ, Azimullah DE, Mechkarska S, Hasan M, et al. (2010) Cholinergic stimulation of the immune system protects against lethal infection by Salmonella enterica serovar Typhimurium. Immunology, 130: 388-398.

15. Hofer S, Eisenbach C, Lukic IK (2008) Pharmacologic cholinesterase inhibition improves survival in experimental sepsis. Crit Care Med 36: 404-408.

16. Akinci SB, Ulu N, Yondem OZ, Firat P, Guc MO, et al. (2005) Effect of neostigmine on organ injury in murine endotoxemia: missing facts about the cholinergic antiinflammatory pathway. World J Surg 29: 1483-1489.

17. McGee PL, McGeer E, Rogers J, SibleyJ (1990) Anti-inflammatory drugs and Alzheimer disease. Lancet 335: 1037. 